\title{
Educación de adultos en Chile: percepciones y valoraciones de sus beneficiarios
}

OSCAR ESPINOZA DIAZ

Programa Interdisciplinario de Investigaciones em Educación (PIIE), Santiago, Chile

DANTE CASTILLO GUAJARDO

Programa Interdisciplinario de Investigaciones em Educación (PIIE), Santiago, Chile

LUIS EDUARDO GONZÁLEZ FIEGEHEN

Programa Interdisciplinario de Investigaciones em Educación (PIIE), Santiago, Chile

\section{RESUMEN}

El propósito del artículo es analizar las percepciones de los beneficiarios de la principal modalidad de educación de adultos en Chile respecto de su experiencia educativa. El estudio se basa en un enfoque empírico analítico, y diseño no experimental. La muestra cuantitativa se obtuvo por conglomerados representativos de centros educativos de Santiago de Chile y permitió encuestar a 252 individuos que asisten a la modalidad regular. La muestra cualitativa consideró 30 estudiantes que fueron abordados por la vía de entrevistas estructuradas. Los resultados confirman que esta población proviene de grupos con bajo nivel socioeconómico, con historial de fracaso escolar reiterado, y que la representación del fracaso escolar la asocian a desempeños individuales más que a factores de contexto. Es por ello que el fracaso es percibido como un acto de irresponsabilidad, aun cuando los antecedentes muestran que el fenómeno es multicausal, donde intervienen factores externos e internos al sistema escolar.

PALABRAS CLAVE

educación de adultos; re-escolarización; deserción escolar; Chile. 


\title{
ADULT EDUCATION IN CHILE: PERCEPTIONS AND ASSESSMENTS OF ITS BENEFICIARIES
}

\begin{abstract}
The purpose of this paper is to analyze the perceptions and assessments of the beneficiaries of the main modality of adult education in Chile with regard to their educational experience. The study is based on an analytical empirical approach, and non-experimental design. A quantitative representative sample was obtained by clusters of schools in Santiago de Chile and allowed to survey 252 individuals attending the regular modality. The qualitative sample considered 30 students who were approached through structured interviews. The results confirm that this population comes from lower socioeconomic groups, with a history of repeated academic failure, but also support the conclusion that the representation of school failure is associated with individual performances rather than contextual factors. School failure is presented as an act of personal irresponsibility, even though the antecedents show it is a multicausal phenomenon, where external and internal factors intervene in the school system.
\end{abstract}

KEYWORDS

Adult education; re schooling; drop out; Chile.

\section{EDUCAÇÃO DE ADULTOS NO CHILE: PERCEPÇÃO E AVALIAÇÃO DE SEUS BENEFICIÁRIOS}

\section{RESUMO}

O objetivo do trabalho é analisar as percepções e avaliações dos beneficiários do principal sistema de educação de adultos no que diz respeito à sua experiência educacional. $\mathrm{O}$ estudo baseia-se numa abordagem empírico-analítica de design não experimental. A amostra quantitativa foi obtida por agrupamentos, representativos de centros educativos da cidade de Santiago do Chile, e permitiu o levantamento de 252 casos. A amostra qualitativa considerou 30 alunos que foram abordados por meio de entrevistas estruturadas. Os resultados confirmam que essa população vem de grupos com baixo nível socioeconômico, com um histórico de insucesso escolar repetido, no qual a consequência do fracasso escolar é associada aos desempenhos individuais, em vez de a fatores externos. Por isso o fracasso é assumido como um ato de irresponsabilidade pessoal, mesmo quando os antecedentes mostram que o fenômeno é multicausal, em que intervêm fatores externos e internos ao sistema escolar.

PALAVRAS-CHAVE

educação de adultos; reeducação; abandono; Chile. 


\section{INTRODUCCIÓN ${ }^{1}$}

Los países de la región latinoamericana, y Chile en particular, viven un período de vertiginosas transformaciones sociales, caracterizado por la difusión masiva de las tecnologías de la información y un acelerado proceso de renovación del conocimiento que pone en cuestión los saberes aprendidos (Castells, 2012). Nos vemos así enfrentados al reto continuo de adaptarnos a las exigencias de un mundo en constante cambio, lo que depende en gran medida de la adquisición de nuevos conocimientos que nos permitan contrarrestar las incertidumbres y, como producto de ello, lograr la inclusión y la promoción social (Castillo, Espinoza y González, 2013; Sarrate, 1997). En este escenario, la educación adquiere una importancia fundamental.

Las personas que se encuentran en mayor desventaja frente a los desafíos que se imponen en la actualidad son quienes no han alcanzado un nivel de instrucción elemental, vale decir, un conjunto básico de habilidades y destrezas (lectura, escritura, expresión oral, etc.) que permitan desenvolverse eficazmente en la vida (trabajar con dignidad, participar de la vida cívica, etc.), y que a la vez sirvan como base para seguir adquiriendo nuevos conocimientos, de acuerdo con las necesidades e intereses particulares (Leenders y Veugelers, 2009; Sousa, 2008; Veugelers, 2011). Estos aprendizajes mínimos se asocian a lo que se entrega en la escuela en sus ciclos primario y secundario, lo que no quiere decir que quienes no hayan concluido estos estudios necesariamente carezcan de estas herramientas. La mayoría de las veces la falta de una certificación que acredite haber finalizado la etapa escolar trae consecuencias tremendamente negativas para las personas que se encuentran en esta situación, tanto a nivel personal como social (Espinoza, Castillo y González, 2013a, 2013c; Espinoza et al., 2014).

De este modo, considerando la incertidumbre propia de estos tiempos y los requerimientos y desafíos que implica la actual fase de desarrollo que enfrentan los países de la región y Chile en particular - donde la educación y las calificaciones certificadas cobran cada vez mayor importancia - , se vuelve relevante la denominada "educación de adultos y jóvenes" no escolarizados, en adelante EPJA. ${ }^{2}$ Si bien este concepto es bastante amplio (Merriam y Brockett, 2007), aquí se entiende como aquella modalidad orientada a desarrollar habilidades y contenidos mínimos interrumpidos por procesos de abandono escolar en algún momento de la trayectoria vital de personas que pueden ser calificadas de adultas (Espinoza et al., 2014a). Dicho lo anterior resulta entonces absolutamente necesario plantearse la pregunta respecto de la pertinencia de los programas impartidos en Chile dirigidos a esta población, en vista de su eventual actualización y reorientación, ya sea desde sus aspectos curriculares

1 Los autores agradecen el financiamiento entregado por la Comisión Nacional de Investigación Científica y Tecnológica (CONICYT) a través del Proyecto Fondecyt n. 1121079 titulado "Factores asociados al éxito de los Programas de reinserción educativa de jóvenes desertores del sistema escolar: la evaluación de la experiencia chilena”.

2 En el caso chileno, los programas de educación de adultos incorporan a población menor de 18 años y el Ministerio de Educación lo denomina Educación de Personas Jóvenes y Adultas (EPJA). 
y/o desde sus aspectos organizativos y administrativos. En la medida en que estos programas sean pertinentes - vale decir, que respondan efectivamente a los cambios de la sociedad y a las características de sus estudiantes -, ellos estarán entregando los conocimientos y habilidades necesarios para el mejoramiento de las condiciones materiales de vida, así como de la autoestima de una parte importante de la población chilena que no ha concluido sus estudios primarios y/o secundarios.

En términos generales, en la adultez la no-escolaridad y la escolaridad interrumpida son situaciones particularmente sensibles para los individuos afectados, que suelen ser vividas en términos de fracaso personal y estar asociadas a situaciones personales, familiares o sociales ligadas a condiciones de mayor vulnerabilidad. La re-escolarización de la población que vio interrumpidos sus estudios tiende a ser percibida por los propios participantes de los distintos programas como un medio que ayudaría a mitigar algunos de los factores de exclusión dándoles nuevas posibilidades, es decir, contribuyendo a mejorar sus competencias laborales $-\mathrm{y}$ con ello sus condiciones de vida materiales - así como su autoestima y autovaloración (Espinoza, Castillo y González, 2013b). Por otra parte, las situaciones, experiencias y/o condiciones de vida que en un momento determinado hicieron imposible la continuación de estudios o que promovieron el abandono escolar son de carácter muy diverso (Rumberger, 2001).

En Chile actualmente son tres las modalidades de regularización de estudios o de "segunda oportunidad", que atienden a jóvenes y adultos que por diversas circunstancias no finalizaron la educación primaria y/o secundaria (MINEDUC, 2012). Estas son la "regular", que consiste en una modalidad de estudios especialmente dirigida a personas jóvenes y adultas que, por distintas razones, no iniciaron o no completaron su proceso escolar. Consta de educación básica y educación media, niveles escolares que se realizan en un tiempo menor a lo establecido para la educación formal de niños y jóvenes. Los estudios cursados a través de esta modalidad son certificados por el establecimiento educacional y son válidos para todos los efectos legales. La licencia de enseñanza media de educación de adultos acredita que se han completado los estudios medios y que la persona puede continuar estudios superiores; la "flexible", que consiste en una oferta semi-presencial dirigida a personas jóvenes y adultas, que se caracteriza por ajustarse a las necesidades de los estudiantes trabajadores, y; la denominada "proyectos de reinserción escolar", que consiste en una modalidad que atiende a estudiantes menores de 14 años de edad, que están fuera del sistema escolar, sobre la base de una oferta gestionada por instituciones públicas y privadas que presentan un proyecto a un fondo concursable administrado por el Ministerio de Educación (MINEDUC). De todas ellas, la primera es la más importante por contar con el mayor número de beneficiarios. Acometer la evaluación de este tipo de modalidades no es tarea fácil debido a la singularidad de las instituciones y experiencias dirigidas a las personas adultas, sin embargo, es urgente pues la evaluación es inherente a la consecución de la calidad y mejora de los procesos de enseñanza-aprendizaje (Sarrate, 1997). Así, y como un primer paso hacia la evaluación, el objetivo principal de este estudio es indagar en los perfiles y en las experiencias de los estudiantes de la principal modalidad de educación de jóvenes y adultos existente en Chile, esto es, la modalidad regular. 
El artículo se ha organizado en cinco partes. En primer lugar, se provee información general acerca de la modalidad regular. En segundo lugar, se aportan antecedentes teóricos relativos a la deserción escolar y a los conceptos de educación de jóvenes y adultos y re-escolarización. Luego se expone la metodología que sirvió de base para el estudio, para posteriormente presentar los principales resultados tanto desde un punto de vista cuantitativo (análisis descriptivo de datos compilados tras la aplicación de una encuesta) como cualitativo (análisis de contenido de entrevistas). Finalmente, se presenta un apartado con las principales conclusiones derivadas de la investigación.

\section{ANTECEDENTES CONCEPTUALES}

\section{LA MODALIDAD REGULAR DE EDUCACIÓN DE ADULTOS EN CHILE}

Recién en el año 2003 en Chile se estableció la obligatoriedad de la educación secundaria, ampliando a 12 años el período de educación obligatoria garantizada por el Estado, que hasta esa fecha era de 8 años. ${ }^{3}$ Mientras en 1990 la escolaridad promedio en Chile era de nueve años (6,1 rural y 9,6 urbana), desde el año 2000 a la fecha esta se ha ido estabilizando en torno de los 10 años (Mideplan, 2011). Este comportamiento se explica, en gran medida, por la estabilización de la tasa de asistencia neta en la educación primaria por sobre el 90\% desde 1990, y en educación secundaria por sobre el 70\% desde el año 2003 (MIDEPLAN, 2011).

Hacia el año 2008 poco más de la mitad de la población mayor de 25 años había egresado de la educación secundaria, mientras que en el segmento de 18 a 34 años este porcentaje era superior al 90\% (MINEDUC, 2008), tendencia que se ha mantenido hasta el día de hoy (Espinoza et al., 2014). Dichas cifras dan cuenta de dos realidades disímiles. Por un lado, la existencia de un gran volumen de población adulta que no completó la educación secundaria cuando no era obligatoria.Por otro, la existencia de un segmento de población adolescente excluida del sistema escolar. En la actualidad en Chile existirían más de 100 mil menores en edad escolar que no estarían asistiendo a la escuela (MINEDUC, 2013).

Para enfrentar situaciones como las antes señaladas, el sistema educativo chileno dispone de diferentes modalidades y programas de re-escolarización. En este contexto, la principal modalidad con la que cuenta el sistema para permitir a las personas mayores de 18 años — sin escolaridad o con escolaridad incompleta iniciar, continuar o completar su formación escolar interrumpida corresponde a la denominada "regular". La modalidad regular permite completar los estudios de educación primaria en solo tres años, y los de educación secundaria en dos. ${ }^{4}$

Considerando la dificultad de estudiar en horarios regulares, la modalidad se lleva a cabo a través de las llamadas Terceras Jornadas, las cuales corresponden

3 Hasta esa fecha, en Chile solo era obligatoria la educación básica (primaria). En 1920 eran obligatorios cuatro años de escolaridad, seis años en 1929 y ocho años desde 1965.

4 En Chile la educación primaria consta de ocho niveles (primero a octavo básico), mientras que la educación secundaria de cuatro (primero a cuarto medio). 
a la jornada vespertina de los diversos establecimientos educacionales que durante el día atienden a niños y jóvenes sin problemas de escolarización. Paralelamente, todas aquellas personas que poseen tiempo a disposición durante el día para estudiar pueden iniciar o completar sus estudios en los Centros Integrados de Educación de Adultos (CEIA). Estos centros son establecimientos educacionales dedicados exclusivamente a la atención de jóvenes y adultos. Para acceder como beneficiario al sistema se requiere únicamente acreditar la mayoría de edad (18 años cumplidos) y documentar el último nivel de enseñanza aprobado.

\section{INTERRUPCIÓN DE LA ESCOLARIZACIÓN}

La interrupción de la escolarización - tradicionalmente asimilada bajo el concepto de deserción escolar - se puede caracterizar como un proceso de alejamiento y de abandono paulatino de un espacio cotidiano - como es la escuela - que implica también el abandono de ciertos ritos personales y familiares que inciden en el desarrollo de la identidad y la proyección personal de un individuo (Comisión Intersectorial de Reinserción Educativa, 2006; Espinoza et al., 2010; Espinoza et al., 2012; Espinoza, Gonzalez e Loyola, 2012).

Los factores que originan la deserción escolar se suelen agrupar en dos grandes marcos interpretativos, cuyo énfasis está puesto en variables de índole extraescolar e intraescolar, respectivamente. Entre los factores extraescolares se identifica a la situación socioeconómica y al contexto familiar como las principales causales del abandono escolar. Se mencionan la pobreza y la marginalidad, la búsqueda de trabajo, la disfuncionalidad familiar y las bajas expectativas de la familia con respecto a la educación, entre otros desencadenantes (Castillo, 2003; Croninger y Lee, 2001; Pomerantz, Moorman y Litwack, 2007; PREAL, 2003).

Entre los resultados más claros y consistentes que ha arrojado la investigación empírica sobre el problema de la deserción escolar (en el nivel primario y secundario) se ha establecido un fuerte vínculo entre el abandono y el estatus socioeconómico, medido este último normalmente a partir del nivel educacional de los padres y del ingreso del grupo familiar (Rumberger, 2001). Diversos estudios empíricos han demostrado que los estudiantes que provienen de familias de bajo estatus socioeconómico presentan mayores probabilidades de desertar del sistema escolar que aquellos insertos en familias de estatus socioeconómico medio y alto (Bryk y Thum, 1989; Goldschmidt y Wang, 1999; Ingrum, 2007; Janosz et al., 1997; Rumberger, 2011; Rumberger y Thomas, 2000; Rumberger, 1995; Rumberger, 1983; Santos, 2009).

El vínculo entre el contexto familiar, la condición de vulnerabilidad y la deserción escolar puede visualizarse a través del concepto de capital cultural (Bourdieu, 1997) que postula que las diferencias de capital cultural, cuando se compara el apoyo escolar que reciben los alumnos de parte de las familias al momento de asistir a clases, no solo se traducen en un buen rendimiento académico, sino que también en un apoyo implícito a la motivación y valoración de la educación, disminuyendo los factores de abandono. En este sentido, algunos estudios muestran que la probabilidad de permanecer en el sistema educacional, junto a los factores asociados al nivel de ingreso familiar, está relacionada en gran medida con la actitud que se 
genera a partir del clima educacional que produce la familia (Espinoza et al., 2012; Mike, Nakajjo y Isoke, 2008; Rumberger, 2011; Rumberger y Lim, 2008).

El fenómeno de la vulnerabilidad y la deserción escolar también se desencadena por razones económicas: está unido a la necesidad de ingresar tempranamente al mundo laboral, para de esta forma satisfacer los requerimientos económicos familiares (McNeal, 1997; Perreira, Harris y Lee, 2006; Warren y Cataldi, 2006; Warren y Lee, 2003). Si bien la inserción laboral temprana puede significar beneficios económicos inmediatos para quienes dejan la escuela, lo cierto es que la interrupción de los estudios escolares a mediano y largo plazo incide en las oportunidades de bienestar, por cuanto los desertores por lo general presentan una situación laboral más precaria y niveles de renta más bajos (Espíndola y León, 2002; Rotermund, 2007; Rumberger, 2011).

Por otra parte, dentro de los factores intraescolares se señalan los problemas conductuales, el bajo rendimiento académico, el autoritarismo docente y el adultocentrismo, entre otros elementos, como las principales causales que detonan la deserción temprana desde el sistema (Espinoza et al., 2010; Espinoza et al., 2012; Rumberger y Lim, 2008; Marshall, 2003). Diversos estudios plantean que la escuela "fabrica" el fracaso escolar para muchos de sus jóvenes. Con esto se quiere indicar, por cierto, que la pérdida del valor atribuido a la asistencia y permanencia en un establecimiento educativo también se relaciona con lo que ocurre dentro de la propia escuela. No son solo los jóvenes los que por su desarrollo personal pierden el interés por asistir a la escuela, sino que esta también de alguna manera los "expulsa" (Espinoza et al., 2012; Espinoza et al., 2012; Raczynski, 2002; Rumberger, 2001; Schkolnik y Del Río, 2002).

Junto con estos factores de orden intraescolar existen otros más vinculados a las propias conductas de los estudiantes al interior de la escuela. Como determinantes de mayores tasas de deserción escolar se mencionan aquí ciertos comportamientos, tales como: el ausentismo y la baja participación en actividades extracurriculares ( $\mathrm{McNeal}$, 1995; Yin y Moore, 2004) — ambos indicadores de un escaso compromiso con la vida escolar -,y el mal comportamiento y las malas relaciones con profesores y compañeros en la escuela (Cairns, Cairns y Neckerman, 1989; Ou et al., 2007).

Más allá de los factores que explican la interrupción de la escolarización en cada caso particular — sean extra o intraescolares, o una combinación de ambos quienes no han concluido la etapa escolar generalmente se encuentran en una situación de mayor vulnerabilidad, asociada a empleos precarios, de baja calificación y peor remunerados, además de sufrir la estigmatización producto de este hecho, con todas sus consecuencias desde el punto de vista de la autopercepción y la autoestima.

\section{LA EDUCACIÓN DE ADULTOS}

El sistema educativo chileno contempla distintas modalidades y programas de re-escolarización dentro del marco de la educación de adultos - entre ellos la modalidad regular, la de mayor cobertura nacional - para aquellas personas que en algún momento de su trayectoria vital abandonaron la escuela, ya sea por algunos de los motivos antes señalados u otros. 
Cabe señalar que al hablar de educación de adultos surge la pregunta respecto de qué es lo que define esta condición. Merriam y Brockett $(1996,2007)$ señalan que la adultez puede ser entendida como una construcción sociocultural. En este sentido, la respuesta a la pregunta acerca de quién puede ser considerado un "adulto" es construida por una sociedad y cultura particular en un momento determinado. Estos autores apuntan que los criterios que se utilizan para delimitar la etapa adulta son variados. En términos biológicos, por ejemplo, muchas culturas consideran la pubertad como la puerta de entrada a la adultez. Por otra parte, la definición legal enfatiza la edad cronológica, mientras que otras conceptualizaciones ponen el acento en la madurez psicológica o en los roles sociales que desempeñan las diferentes personas. Smith (1999) sostiene que la edad por sí misma no es criterio suficiente para definir la adultez, ya que para que una persona sea considerada como adulta esta debe auto-reconocerse como tal, ello producto de su estado biológico o legal, de su estado psicológico o de sus formas de comportamiento o roles sociales.

Más allá de la controversia acerca de quién puede o no ser considerado un adulto, Merriam y Brockett (2007) postulan que todas las definiciones de educación de adultos usualmente incluyen referencias a dos elementos:

1. al estatus "adulto" de los estudiantes, en función a su condición etaria, y

2. a la noción de que este campo abarca actividades de carácter planificado.

Para estos autores la educación de adultos incluye todas aquellas actividades diseñadas y planificadas con el propósito de promover el aprendizaje entre aquellas personas cuya edad, roles sociales o su propia auto-percepción los define como tales. En el caso chileno, para efectos de políticas públicas, prevalece el criterio etario a la hora de definir quién cae dentro de la categoría de adulto: es una persona adulta aquella que tiene 18 o más años de edad, lo que al mismo tiempo constituye un requisito de ingreso para algunos programas de re escolarización (Espinoza, Castillo y González, 2013a, 2013b, 2013c; MINEDUC, 2012). Ahora bien, esta definición legal puede estar asociada, aunque no necesariamente, a otros elementos definitorios de la adultez como son los roles sociales -por ejemplo, el que una persona se encuentre inserta en el mundo laboral, o que tenga hijos-, aspectos identitarios, etc.

Establecer qué se entiende por educación de adultos no solo plantea dificultades desde el punto de vista de qué se entiende por población adulta, sino que también en cuanto a cuáles son los tipos de actividades planificadas específicas que caen dentro de este campo. Existe consenso en que se trata de actividades de carácter voluntario dirigidas a un segmento particular — la población adulta, más allá de cómo se defina esta categoría - y en cuanto tales sus objetivos y las configuraciones en que se desarrollan es muy vasto (Zoellick, 2009). En este sentido, cabe destacar que en el campo de la educación de adultos han surgido denominaciones tan variadas como educación vitalicia, educación continua, educación compensatoria, educación para la tercera edad, etc. (OECD, 2001, 2004; Schuller y Watson, 2009; UNESCO, 2009a, 2009b). Estos rótulos mantienen relación y son utilizados para designar diferentes áreas de la educación de adultos. Una definición ampliamente aceptada de educación de adultos es la que provee UNESCO (1976, p. 124) que plantea: 
La expresión "educación de adultos" designa la totalidad de los procesos organizados de educación, sea cual sea el contenido, el nivel o el método, sean formales o no formales, ya sea que prolonguen o reemplacen la educación inicial dispensada en las escuelas y universidades, y en forma de aprendizaje profesional, gracias a las cuales las personas consideradas como adultos por la sociedad a la que pertenecen, desarrollan sus aptitudes, enriquecen sus conocimientos, mejoran sus competencias técnicas o profesionales o les dan una nueva orientación, y hacen evolucionar sus actitudes o su comportamiento en la doble perspectiva de un enriquecimiento integral del hombre y una participación en un desarrollo socioeconómico y cultural equilibrado e independiente.

Sarrate (1997) extrae algunas consideraciones de esta definición, destacando que tiene un carácter global, abarcando un amplio conjunto de procesos educativos y actividades - con el requisito de que deben estar organizadas - y admitiendo, asimismo, una pluralidad de métodos y modalidades en función de objetivos y demandas formativas - y por ende englobando la totalidad de niveles e incluyendo una amplia gama de contenidos - . Así, la definición de UNESCO da cuenta del programa de re-escolarización que es materia específica de este estudio. Tal como señala Sarrate, dicha definición se vincula con la democratización educativa y cultural por cuanto incluye los programas de "segunda oportunidad" para todos aquellos que no han podido completar la educación primaria o secundaria, y en la práctica los destinatarios a los que se refiere - los adultos — son aquellos que se encuentran en edad post-escolar y han abandonado el sistema formal.

Los programas de re-escolarización, como modalidad específica de la educación de adultos, están dirigidos a una población particular: aquellas personas adultas que no han completado sus estudios primarios y/o secundarios por haber desertado del sistema escolar formal en algún momento de sus vidas. Merriam y Brockett (2007) señalan que las distintas modalidades de educación de adultos se pueden diferenciar en función de los objetivos y necesidades que cubren. Así, los programas de re-escolarización caben dentro de las iniciativas cuya función puede ser calificada de "remedial" o "compensatoria", por cuanto están orientados a desarrollar habilidades y contenidos mínimos interrumpidos en algún momento de la trayectoria vital de los destinatarios.

Ahora bien, más allá de los objetivos específicos de los diferentes tipos de programas, Sarrate (1997) apunta que en términos generales se entiende que la meta de la educación de adultos es la formación integral de las personas a través del desarrollo de aptitudes, la adquisición de conocimientos y la mejora de competencias técnicas y profesionales, de modo de posibilitar su mejor inserción social, así como propiciar la autorrealización personal y la participación social.

Un aspecto muy importante respecto de los estudiantes adultos que no puede ser obviado es que esta población se encuentra motivada para aprender tanto por factores internos como externos, siendo los primeros los que tendrían un mayor peso en el caso de ellos. Las motivaciones externas se vinculan a menudo con los motivos "racionales" que llevan a los adultos a insertarse en un contexto educativo; por ejemplo, en el caso de los programas de re-escolarización, una motivación externa podría ser el conseguir el certificado de enseñanza secundaria que even- 
tualmente permitiría acceder a un mejor puesto de trabajo y, por ende, a un salario más alto. Por otra parte, las motivaciones internas tienen que ver más bien con la autorrealización y la autoestima, factores que podrían explicar el porqué de ingresar a programas de re-escolarización para terminar estudios que en algún punto de la vida de los adultos quedaron inconclusos (Espinoza et al., 2014a, 2014b; Knowles, 1980).

\section{METODOLOGÍA}

El artículo se enmarca en una investigación de carácter mixto que considera un diseño no experimental de corte transversal. La información que sirve de base para el análisis proviene de una encuesta ${ }^{5}$ aplicada en forma presencial - entre los meses de octubre de 2012 y enero de 2013 - a una muestra de 523 estudiantes que cursan alguna de los tres programas de re-escolarización (regular, flexible y proyectos de reinserción escolar) en la ciudad de Santiago de Chile, repartidas en un total de 23 instituciones educativas. No obstante, el presente artículo analiza las declaraciones de 252 estudiantes que correspondieron al estrato de los estudiantes de la modalidad EPJA-Regular. La muestra cualitativa se obtuvo del grupo de estudiantes que se entrevistó, en virtud de una representación cualitativa estructural, es decir, incluyendo casos que mostraran la heterogeneidad de perfiles de estudiantes. En las entrevistas en profundidad participaron 12 estudiantes EPJA-Regular.

La muestra se obtuvo de manera probabilística mediante la técnica de muestreo por conglomerados, y el marco muestral se construyó a partir del total de registros de matrículas provisto por la Coordinación Nacional de Educación de Personas Jóvenes y Adultas, del MINEDUC.

\section{RESULTADOS}

\section{PERFIL DE LOS ESTUDIANTES DE LA EDUCACIÓN DE ADULTOS DE LA MODALIDAD REGULAR}

En este apartado se presentan los principales atributos y características de los estudiantes que están asistiendo al programa de educación de adultos de la modalidad regular dispuesto por el MINEDUC. Es así como, desde el punto de vista de la condición de género, se observa que la mayor parte de los estudiantes que concurren a la modalidad regular son de sexo femenino $(64,5 \%)$, es decir, prácticamente dos de cada tres asistentes. Del mismo modo, si el promedio de edad de la muestra es de 28,12 años, la media de las mujeres es superior por 6,35 años respecto de la registrada en el caso de los hombres (Tabla 1). Lo interesante de esta información,

5 Para la construcción del cuestionario se empleó como referente validado el instrumento elaborado por el Instituto Nacional de la Juventud (INJ) y el Ministerio de Desarrollo Social (http://www.injuv.gob.cl). El instrumento en cuestión se titula "Cuestionario de la Sexta Encuesta Nacional de Juventud”. Esta encuesta nacional es un instrumento de análisis cuantitativo que se aplica desde 1994, cada tres años, con la finalidad de actualizar el diagnóstico nacional en juventud, a partir de aproximadamente 100 ítems orientados a medir educación, trabajo, participación social, representaciones valóricas y discriminación. 
podría estar asociada a la capacidad que tendrían las estudiantes de género femenino para adaptarse y sobreponerse a los ritos y cultura de los establecimientos educativos y, quizás a los obstáculos socioculturales que la sociedad chilena le "impone" al rol femenino, en cuanto a postergar sus expectativas educacionales. Con todo, en esta tabla se aprecia que el promedio de edad en total y particularmente en el caso de los hombres tiende a ser bastante menor que el observado en el caso de las mujeres.

Por otra parte, poco más de la mitad de los encuestados $(51,6 \%)$ declara tener hijos (Tabla 2), lo que es reflejo de una responsabilidad familiar que tiene un grupo de estos estudiantes. Es decir, un tipo de responsabilidad asociada a un rol de "adulto" propiamente tal (Smith, 1999). Esta condición permite al mismo tiempo dar cuenta de dos tipologías de estudiantes, a saber: quienes tienen y no tienen hijos. Situación que también permite dar cuenta de los cambios que está experimentando el beneficiario que actualmente ingresa a esta oferta educativa de segunda oportunidad. Lo anterior podría explicarse por la disminución etaria que este tipo de oferta educativa ha estado experimentando en los últimos años.

Un porcentaje algo menor al 50\% (46,7\%) de los encuestados declara tener trabajo, lo que revela la presencia de una alta tasa de desempleo entre los estudiantes de la EPJA Regular, quizás en parte por la dificultad de encontrar trabajo por el hecho de no poseer la certificación que acredita la finalización de la etapa escolar (Tabla 3).

Tabla 1 - Estudiantes según sexo y promedio de edad (2013)

\begin{tabular}{l|c|c}
\hline Sexo & Porcentaje & Promedio de edad \\
\hline Femenino & 64,5 & 30,36 años \\
\hline Masculino & 35,5 & 24,01 años \\
\hline Total & 100 & 28,12 años \\
\hline
\end{tabular}

Fuente: Banco de dados de la encuesta.

Elaboração por la autores.

Tabla 2 - Estudiantes según tenencia de hijos

\begin{tabular}{l|c}
\hline Hijos & $\%$ \\
\hline Tiene hijos & 51,6 \\
\hline No tiene hijos & 48,4 \\
\hline Total & 100 \\
\hline
\end{tabular}

Fuente: Banco de dados de la encuesta.

Elaboração por la autores.

Tabla 3 - Estudiantes según condición laboral (2013)

\begin{tabular}{l|c}
\hline Condición laboral & $\%$ \\
\hline Trabaja & 46,7 \\
\hline No trabaja & 53,3 \\
\hline Total & 100 \\
\hline
\end{tabular}

Fuente: Banco de dados de la encuesta.

Elaboração por la autores. 
Pero al mismo tiempo, esta condición de no trabajador, igualmente está explicando el giro que está teniendo la oferta ministerial para la educación de adultos y jóvenes, dado que actualmente está aumentando la oferta de cursos diurnos y disminuyendo la oferta en horarios vespertinos.

Junto a lo anterior, también se aprecia que, del total de quienes declaran encontrarse trabajando la mayor parte lo hace con jornadas laborales mayores a medio día $(61,2 \%)$ y con contrato de trabajo formal (58\%). Pero, prácticamente otro $40 \%$ restante señaló que solo trabaja medio día, cifra que incluye a un grupo de alumnos que señalen trabajar por horas $(24,1 \%)$. El dato anterior, agregado al hecho que un porcentaje importante declaró trabajar sin contrato $(38,7 \%)$, revela que, por una parte, existe un importante grado de precariedad en la situación laboral de muchos de ellos, pero al mismo tiempo permite apreciar que la exigencia laboral tiene menos presencia que en décadas pasadas (Tablas 4 y 5 ).

Al observar la Tabla 6 destaca el hecho que el 84,9\% de los encuestados abandonó los estudios en algún nivel del ciclo primario. De este porcentaje, el 26,8\% de los estudiantes encuestados señaló que había dejado la escuela en el último año de primaria. Esta información es bastante relevante en la medida que confirma que el año de transición entre la escuela primaria y secundaria, marca la cumbre del abandono o deserción escolar. Con todo lo más destacado, es el hecho que para esta modalidad los estudiantes provienen principalmente de aquella población que no completó la enseñanza primaria.

Confirmando lo que señalan diversos estudios empíricos (Espíndola y León, 2002; Espinoza et al., 2012), a saber, que existe una relación entre el nivel de escolaridad de la madre y una mayor posibilidad de haber desertado, se observa en la

Tabla 4 - Estudiantes según tipo de jornada laboral (2013)

\begin{tabular}{l|c}
\hline Jornada laboral & $\%$ \\
\hline Más de medio día & 61,2 \\
\hline Medio día & 14,7 \\
\hline Por horas & 24,1 \\
\hline Total & 100 \\
\hline
\end{tabular}

Fuente: Banco de dados de la encuesta.

Elaboração por la autores.

Tabla 5 - Estudiantes según tipo de relación laboral (2013)

\begin{tabular}{l|c}
\hline Relación laboral & $\%$ \\
\hline Con contrato & 58,0 \\
\hline Sin contrato & 38,7 \\
\hline No sabe & 3,3 \\
\hline Total & 100 \\
\hline
\end{tabular}

Fuente: Banco de dados de la encuesta.

Elaboração por la autores. 
Tabla 7 que el 74,4\% de las madres de los estudiantes de los cursos EPJA regular, no completó la enseñanza media, vale decir, no finalizó su etapa escolar. De este modo, los alumnos encuestados que participan de la modalidad regular provienen de familias de bajo capital cultural y donde existe un historial de deserción, por eso no es extraño que presenten itinerarios educativos similares a los de sus madres (Tabla 7).

Tabla 6 - Estudiantes según último nivel escolar aprobado antes de integrarse al programa (2013)

\begin{tabular}{l|c}
\hline Último nivel aprobado & $\%$ \\
\hline Nunca asistió & 0,9 \\
\hline $1^{\circ}$ Primaria & 6,8 \\
\hline $2^{\circ}$ Primaria & 18,2 \\
\hline $3^{\circ}$ Primaria & 5,0 \\
\hline $4^{\circ}$ Primaria & 14,5 \\
\hline $5^{\circ}$ Primaria & 3,6 \\
\hline $6^{\circ}$ Primaria & 6,4 \\
\hline $7^{\circ}$ Primaria & 3,6 \\
\hline $8^{\circ}$ Primaria & 26,8 \\
\hline $1^{\circ}$ Secundaria & 2,3 \\
\hline $2^{\circ}$ Secundaria & 7,7 \\
\hline $3^{\circ}$ Secundaria & 4,1 \\
\hline $4^{\circ}$ Secundaria & 0,0 \\
\hline Total & 100 \\
\hline
\end{tabular}

Fuente: Banco de dados de la encuesta.

Elaboração por la autores.

Tabla 7 - Estudiantes según nivel educacional alcanzado por la madre (2013)

\begin{tabular}{l|c}
\hline Nivel educacional alcanzado por la madre & $\%$ \\
\hline No fue a la escuela & 10,6 \\
\hline Básica incompleta & 26,4 \\
\hline Básica completa & 18,7 \\
\hline Media incompleta & 18,7 \\
\hline Media completa & 20,3 \\
\hline Superior incompleta & 1,6 \\
\hline Superior completa & 3,7 \\
\hline Total & 100 \\
\hline
\end{tabular}

Fuente: Banco de dados de la encuesta.

Elaboração por la autores. 
Por último, llama la atención que para un porcentaje importante de los encuestados (30\%) esta no es la primera vez que participa de un curso de similares características. Este dato puede estar mostrando, por una parte, las ansias que muchas de estas personas tienen por concluir sus estudios primarios y/o secundarios (Tabla 8). Pero los datos también muestran que un porcentaje importante de esta población (30\%) indicó haber participado de esta experiencia educativa lo que estaría evidenciando la presencia de fracasos educativos recurrentes o de una deserción crónica. Considerando el tipo de pregunta incorporada en el cuestionario empleado, el hecho que un estudiante declare que no es la primera vez que participa en un programa de re-escolarización, significa que ya participó, pero que abandonó prematuramente este proceso. En otras palabras, ya sea por motivos externos y/o internos a la institución escolar, un 30\% de los participantes de la modalidad regular, no han finalizado los cursos de educación de adultos, en un primer momento.

\section{PERCEPCIONES DE LOS BENEFICIARIOS SOBRE EL FRACASO ESCOLAR}

En general, el fracaso escolar puede considerarse como cúmulo de resultados negativos que inciden en el desempeño y la trayectoria escolar de los estudiantes, que al ser constantes y reiterados, inducen situaciones de abandono y deserción escolar. Como resultado, cada persona tiende a buscar explicaciones o causas que revelen las situaciones en las que se ven envueltos cotidianamente. En el caso de la deserción escolar, cognitivamente, cada sujeto intenta reconstruir integral y significativamente su propia experiencia, ya sea atribuyendo causas a la propia acción personal o la de otro.

En consideración a lo anterior, en el presente apartado se analizan las opiniones de los estudiantes que asisten a estos programas de educación de adultos respecto de las motivaciones que tuvieron para desertar del sistema escolar formal. Estas opiniones se agruparon en dos categorías: factores personales y situacionales. Concretamente, como factores personales se entenderá a aquellas representaciones de la deserción o fracaso escolar, declaradas por los estudiantes, que residen principalmente en la propia persona (internas al sujeto), situándose en sus habilidades, potencialidades, capacidades, motivaciones, esfuerzos y necesidades. En cambio, por factores situacionales se entenderá a aquellas acciones que residen en el ambiente (externas al sujeto), las que comprenden características propias a cada situación, como también así, las consecuencias derivadas del azar o la suerte.

Tabla 8 - Estudiantes matriculados según participación en cursos similares (2013)

\begin{tabular}{l|c}
\hline Participación en cursos similares & $\%$ \\
\hline Sí & 30 \\
\hline No & 70 \\
\hline Total & 100 \\
\hline
\end{tabular}

Fuente: Banco de dados de la encuesta.

Elaboração por la autores. 
Para realizar la distinción antes señalada, se utilizaron las declaraciones manifestadas por los estudiantes en una "pregunta abierta" incluida en el cuestionario usado para la aplicación de la encuesta metropolitana. Esta pregunta sintetiza el motivo declarado (agrupado en factores personales y situacionales) por los estudiantes EPJA, que les impidió continuar estudiando en la educación regular formal (Cuadro 1).

Ahora bien, el Gráfico 1, permite apreciar los resultados del agrupamiento realizado, según el tipo de factores declarados por los beneficiarios. De esta manera,

Cuadro 1 - Tipos de factores asociados a la deserción escolar (2013)

\begin{tabular}{|l|l|}
\hline \multicolumn{2}{|c|}{ Factores Personales Asociados al Fracaso Escolar } \\
\hline - Flojera & - Por salir a carretear \\
- Motivos personales & - Porque repetí \\
- Fui padre & - Desmotivación \\
- Quise ser madre joven & - Pocos recursos destiné al tema \\
- Preferí cuidar a mis hijos & - No me gustaba \\
- No me gusta estudiar & - No quise \\
- Problemas personales & - Por flojo \\
- Por mi rebeldía & - Problemas de conducta míos \\
\hline \multicolumn{2}{|c|}{ Factores Situacionales Asociados al Fracaso Escolar } \\
\hline - Motivos económicos & - Profesores le tenían mala \\
- No lo mandaban al colegio & - Por bullying \\
- Falta de recursos & - Problemas con la escuela \\
- Los médicos se lo prohibieron & - Por fuerza mayor \\
- No pudieron pagar sus padres & - Divorcio de mis padres \\
- Tratamiento médico & - Modelo económico imperante \\
- Cambio de casa & - Por distancia \\
- Me echaron del colegio & - No me dejó mi madrastra \\
\hline
\end{tabular}

Fuente: Banco de dados de la encuesta.

Elaboração por la autores.

Gráfico 1 - Factores declarados asociados a la deserción escolar de la educación formal (2013)

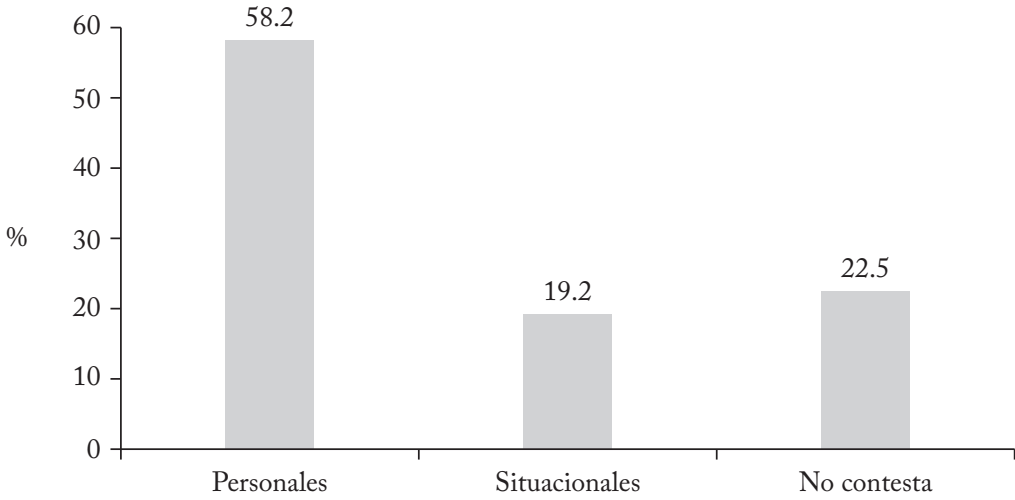

Fuente: Banco de dados de la encuesta.

Elaboração por la autores. 
prácticamente un 60\% del total de estudiantes que asisten a los cursos EPJA, atributen a una decisión personal el principal motivo que les impidió seguir estudiando en el itinerario escolar "normalizado" o "formal". En otras palabras, casi la mitad de los estudiantes EPJA hace una reconstrucción o representación del fracaso escolar, asociable a sus acciones individuales, más que a limitaciones u obstáculos de contexto. La relevancia de este hallazgo, dice relación con la necesidad de profundizar en esta temática, dada la importancia que puede tener para los planes y programas de estudio, el hecho que los estudiantes EPJA, se refieran a sus trayectorias escolares, atribuyéndose la responsabilidad o centrándola en factores exclusivamente individuales. Lo anterior, debido a los eventuales problemas de autoimagen o autoestima y, al efecto que esta imagen pueda tener en el éxito o fracaso que esta población puede experimentar en los cursos de educación de adultos o de segunda oportunidad. Complementariamente, al incluir la condición de género en el análisis, la prueba de "chi-cuadrado" $(0,162)$, no permite señalar que existiesen diferencias estadísticamente significativas entre hombres y mujeres. Por lo mismo, la tendencia anteriormente descrita es similar para ambos sexos.

Cuando se consulta por los motivos de una eventual deserción de los cursos EPJA, alrededor del noventa por ciento de la población encuestada, señala que este escenario se explicaría por motivaciones personales y no situacionales, tal como aconteció en su paso por el sistema escolar formal (Gráfico 2). Esta interpretación dada por los estudiantes es relevante cuando se quiere construir una estrategia para disminuir la deserción y el fracaso educativo en los cursos EPJA. Ello, en la medida que las representaciones que va construyendo esta población sobre sus itinerarios escolares, puede traducirse en una disminución progresiva de la autoestima o en una exacerbación de las actitudes auto-flagelantes. Del mismo modo, el énfasis en factores individuales, tiende a exculpar u omitir los factores asociados a los propios

\section{Gráfico 2 - Factores declarados sobre una eventual futura deserción de los cursos EPJA}

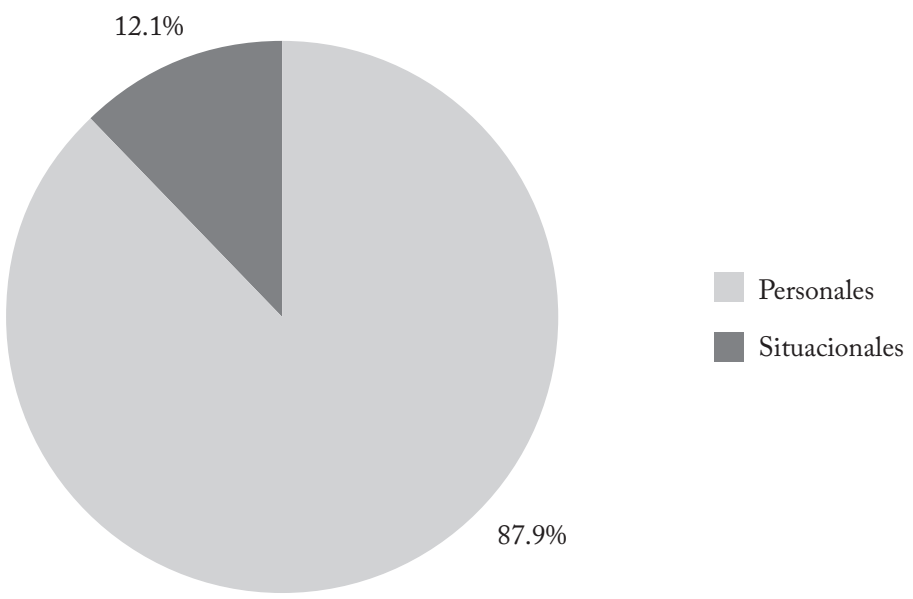

Fuente: Banco de dados de la encuesta.

Elaboração por la autores. 
centros educativos, a la política educativa nacional o a las condiciones sociales en las que se ubica esta población.

\section{REPRESENTACIONES DE LA EXPERIENCIA EDUCATIVA EN CURSOS EPJA}

Para ratificar los hallazgos anteriores, se procedió a analizar la información que proporcionaron los beneficiarios de la modalidad regular de educación de adultos que fueron entrevistados, sobre su experiencia educativa en esta modalidad. Se aprecia, en primer término, que las valoraciones son positivas pero tienden a destacar nuevamente aspectos individuales más que colectivos. Se debe considerar que estas declaraciones han sido recabadas de entre los estudiantes que permanecieron y completaron al menos un periodo dentro del curso de EPJA.

La mayor parte de los entrevistados resalta que su participación en este espacio educativo se ha constituido en un proceso de crecimiento personal. Para ellos, ha significado aumentar la confianza en sí mismos y disminuir los sentimientos de discriminación a las personas con las que se relacionan en los distintos ámbitos de su vida. En esa perspectiva, se plantea respecto de su participación en los cursos que su experiencia es: "Positiva, porque uno crece como persona. Uno se siente más importante al ya saber más” (Estudiante EPJA, 2013).

Los estudiantes, en general, hacen una buena valoración de su experiencia en el curso. Declaran que han obtenido buenas notas y que los ha motivado mucho la entrega de premios por sus buenos promedios, asistencia, etc. En el caso de los alumnos de mayor edad, agregan que en esta etapa de su vida ellos "no están para perder el tiempo" y que por eso intentan aprovecharlo al máximo. En otras palabras, sus justificaciones denotan una actitud con rasgos pragmáticos e instrumentales, asociados a estímulos individuales, tal como se advierte en la siguiente cita:

Es que nosotros no queremos perder el tiempo, para nosotros es corto. Yo aprovecho mi tiempo al máximo [...] yo gané tres premios del curso. Mejor alumna, mejor promedio curso... cuando me gradué de octavo. (Estudiante EPJA, 2013)

Pese a lo anterior, también se observa un discurso que apela a lo colectivo, pues los estudiantes también valoran positivamente la relación con sus pares dentro del contexto de su curso. Pero esta percepción es más evidente entre las personas de más edad, quienes aprecian y destacan la posibilidad de compartir, ser aceptados y apoyados por compañeros menores que ellos. Ello se advierte cuando se declara:

Para mí ha sido muy positivo y lo valoro mucho. El compañerismo ha sido lo más positivo, la entrega de los profesores, la comprensión hacia el alumno. No hubo desunión ni desigualdad, ha sido parejo para todos. (Estudiante EPJA, 2013)

En este mismo registro discursivo, la apreciación positiva de los estudiantes hacia sus pares se extiende también hacia sus profesores. En efecto, ellos se sienten acogidos, valorados y respetados por los docentes. La relación con los profesores para muchos estudiantes es uno de los motivos por los que han permanecido en el programa, ya que éstos jugarían un rol motivacional de importancia tal como queda reflejado a continuación: 
Uno rescata esto porque los profesores son súper acogedores. Cuando tú no entiendes ellos te explican, explican y explican hasta que uno entienda. Es súper grato. (Estudiante EPJA, 2013)

No obstante, con recurrencia la representación de sus itinerarios y experiencias educativas, vuelven a centrase en la esfera de lo privado, de lo individual. Es así como, los estudiantes señalan que venían de vivir con el estigma de no haber terminado sus estudios secundarios, y que lo primero que les transmitieron sus profesores fue la confianza en que ellos eran capaces de cambiar ese estigma, tal como se aprecia en la siguiente cita:

Se siente una confianza tremenda al tener realmente un profesor al frente que te dé pero toda la confianza [...] Me siento muy feliz y contento, ni siquiera me quería tomar vacaciones. (Estudiante EPJA, 2013)

Del mismo modo, muchos estudiantes manifiestan que pasar por los cursos de re-escolarización ha contribuido a incrementar su autoestima. Varios sentían que la vida no les iba a dar ninguna oportunidad para mejorar, a pesar de que tenían ganas de hacerlo como queda reflejado en el siguiente testimonio:

[Es importante] tener el orgullo y oportunidad de poder estudiar a esta altura [...] es la oportunidad que esperaba toda mi vida. (Estudiante EPJA, 2013)

También señalaron que:

La vida a veces a uno le cierra muchas puertas y uno piensa que ya se cerraron y no hay nada que hacer... Pero da estas oportunidades que ojalá muchos más pudieran aprovechar. (Estudiante EPJA, 2013)

Pero, lo que también aparece de forma recurrente en el discurso de estos estudiantes, es que la mejora en su condición de escolaridad, dependería de manera privilegiada de sus "decisiones" y acciones individuales, omitiendo, por ejemplo, la "responsabilidad" vinculada a las instituciones educativas. Dicho de otra manera, para comprender el fenómeno de la deserción y del fracaso escolar recurrente, es necesario considerar la relación entre los factores externos (o factores extra institucionales, tales como, el económico, el vocacional, el motivacional, etc.) e internos (o factores intra institucionales), que en general se entrelazan para explicar el éxito o fracaso educativo de estos programas educativos de segunda oportunidad.

En el mismo registro anterior, los estudiantes de mayor edad, destacan la buena relación con sus pares y el apoyo mutuo frente a los distintos desafíos que se les iban planteando en el desarrollo del curso. Estar con jóvenes que se encontraban en una situación similar a la de ellos les permitió compartir su experiencia de vida y sentirse útiles:

Yo me he sentido bien con estos jóvenes y de repente me siento útil cuando me piden ayuda. No es que yo sepa más, pero de repente puedo decirles cuando lo están haciendo mal, me hacen consultas. No es por nada, pero nosotros fuimos 
el grupo que sacamos mejores notas. Entonces les contábamos como hacíamos las cosas. En mi curso la brecha generacional fue cero altibajos. (Estudiante EPJA, 2013)

Lo interesante respecto de este tipo de discurso es que pese a lo declarado, igualmente los estudiantes de mayor edad se ubican discursivamente en una condición “superior", en relación a los más jóvenes. De hecho, los estudiantes de mayor edad señalan que han intentado apoyar a sus compañeros más jóvenes compartiendo con ellos su experiencia de vida y motivándolos a ser responsables y seguir adelante con sus estudios. Estudiar, para ellos, es una oportunidad para mejorar sus condiciones de vida y pensar en la posibilidad de proseguir estudios. En este sentido, algunos reconocen que "han vuelto a soñar", tal como se ilustra a continuación:

Uno siempre le dice a los chicos, uno que ha caminado ya en la vida, pucha que da rabia cuando un muchacho pasa en los trabajos por al lado tuyo y no tienen el espíritu, no tienen nada pero pasan porque tienen un estudio. Eso a uno le da rabia, porque a mí por lo menos me tocó. Yo estuve en un trabajo por años y por el lado mío pasó una persona que venía con título y yo chao para afuera. No valieron los años de trabajo, empeño, experiencia. (Estudiante EPJA, 2013)

Pero, tal como en los relatos anteriormente aludidos, lo destacable de estos discursos, dice relación con el hecho que estos estudiantes aluden también a los atributos personales e individuales, como un factor de promoción educativa.

Los elementos que identifican como motivación para retomar sus estudios son variados, sin embargo, la mayoría están ligados al mundo laboral. Algunos señalan que volver a estudiar significa para ellos tener la posibilidad de acceder a mejores trabajos y a una mejor remuneración. Cuentan que por no haber finalizado la etapa escolar han perdido la posibilidad de acceder a mejores trabajos. Otros señalan que optaron por ingresar a cursos EPJA para obtener una licencia de conducir que les permita trabajar en medios de transporte o en otras áreas donde se requiera algún servicio vinculado al manejo de vehículos. Es común el discurso que justifica la participación en el programa por la necesidad de mejorar sus credenciales en el contexto de un mercado laboral cada vez más competitivo. No contar con la escolarización obligatoria se transforma en una gran limitación en la medida en que en el mercado laboral se castiga a quien no la tiene: "Uno necesita tener los estudios para poder trabajar. Te exigen el cuarto medio. Lo hice más por el tema de emprender y poder trabajar” (Estudiante EPJA, 2013). También hay estudiantes que han retomado los estudios porque tienen la intención de seguir estudiando y así alcanzar una profesión, tal como se evidencia en la siguiente declaración: "Mi sueño, el que quiero cumplir, es ser parvulario" (Estudiante EPJA, 2013).

Los estudiantes con más edad señalan que retomar los estudios para ellos significa la posibilidad de terminar sus días con la certificación escolar que les permita concluir una etapa de su vida que estaba incompleta. En este caso no se advierte la instrumentalidad que se observa en quienes lo hacen para mejorar su empleabilidad. Para ellos existe una razón más vinculada con su identidad y au- 
toestima. Al respecto se manifiesta: "Mi sueño, a esta edad, es terminar el cuarto medio. Con eso me siento tranquilo, conforme y puedo vivir el resto de mis días en paz" (Estudiante EPJA, 2013).

Si bien, como ya se indicó, los profesores, según las percepciones de los estudiantes, juegan un rol fundamental en la motivación que brindan a los estudiantes para continuar con sus estudios, la familia es otro factor relevante. En el caso de los estudiantes mayores es muy importante que sus familias se sientan orgullosas de ellos, ya que consideran que eleva su propia condición de padres, sienten que son más respetados y menos discriminados por sus hijos y el medio en el que se desenvuelven. Sobre el particular se plantea:

En el caso de mis hijos, ellos están muy contentos de que yo pueda terminar mis estudios. Digamos que se eleva la condición del papá a los hijos [...] Uno mismo trata de igualarse delante de ellos. Ya uno cumplió su sueño, en el caso mío de que los chicos sean más que uno. Entonces como ya pasé esa etapa ahora yo quiero subir a la altura de ellos. (Estudiante EPJA, 2013)

Confirmando lo anterior, otro estudiantes postula:

Los míos (hijos) están orgullosos de mí, porque tomé sola la decisión de terminar mis estudios y después de eso voy a estudiar odontología. Así que es importante terminar. (Estudiante EPJA, 2013)

Por otra parte, en lo que concierne a las valoraciones y expectativas de futuro que poseen los estudiantes estas son dispares dependiendo de su edad. Algunos manifiestan que su experiencia en el curso los ha motivado a continuar estudiando, porque se sienten capaces de seguir adelante y hacer realidad su sueño de estudiar una carrera técnica o universitaria que les permita ser profesionales y poder desempeñarse en aquello que estudien. Esta perspectiva de continuación de estudios suele ser, sin embargo, bastante difusa, quedando la mayor parte de las veces en una declaración de intenciones y aspiraciones, más que en la definición concreta de un proyecto personal a futuro, como se ejemplifica a continuación: “[Me gustaría] seguir una carrera, tener una profesión y poder trabajar en lo que uno estudia" (Estudiante EPJA, 2013). Ahora, si bien la cita anterior alude a la inserción laboral, en términos generales, cuando los estudiantes EPJA se pronuncian sobre el futuro, sus declaraciones están más vinculadas a criterios vocacionales que laborales propiamente, tal como puede apreciarse en las siguientes expresiones:

Me gustaría seguir estudiando, porque si uno quiere ser parvulario tiene que seguir estudiando. Me gustaría hacer hartas cosas, me encantan los niños. Me gustaría trabajar ayudando a los niños, ayudar a los abuelitos, a las personas que lo necesitan. (Estudiante EPJA, 2013)

En mi caso no esperan algo, pero sí podría tratar de ver una carrera., Me gustaría pese a la edad. como un desafío personal, seguir una carrera. (Estudiante EPJA, 2013) 
Seguir estudiando, terminar la básica, media y si se puede llegar a la universidad, rico sería. Si me va bien y termino, lo haría. Ahora dispongo del tiempo, ya críe hijos, nietos. Tengo el tiempo como para estudiar. (Estudiante EPJA, 2013)

Del mismo modo, en las declaraciones de los estudiantes se aprecia que valoran la educación como la posibilidad de mejorar sus condiciones de vida y de crecer como personas. Para ellos ha sido importante demostrarse que son capaces de superar satisfactoriamente un curso - lo que los llena de orgullo, así como también a sus familias - y de generar las condiciones para acabar con la discriminación, laboral y social, a la que han estado o expuestos gran parte de su vida, según sus percepciones. Igualmente, destacan que hoy sienten más cercana y accesible la posibilidad de educarse y poder acceder por medio de becas a carreras técnicas o universitarias, tal como se ilustra en el siguiente planteamiento:

En mis años no había posibilidad. El que llegaba a la universidad era porque tenía plata. Ahora no, hay becas, hay ayuda social. Hay incentivos para poder decir ahora se puede. Con cuarto medio ahora se puede estudiar cualquier carrera. (Estudiante EPJA, 2013)

Lo relevante de esta última cita, dice relación con la representación que tienen los estudiantes sobre el éxito educacional, pues en la actualidad, según sus propias palabras, en Chile existen las condiciones y las garantías para que la población que no pudo culminar sus estudios por alguna razón, pueda continuar y finalizar la educación primaria y secundaria en la modalidad regular. Por lo tanto, implícitamente también es posible deducir que si una persona no prosigue y no finaliza la escolaridad obligatoria, es por una situación, rasgo o motivación personal, pero no por una cuestión social o colectiva.

\section{CONCLUSIONES}

A la luz de los resultados es posible señalar que los estudiantes EPJA de la modalidad regular, corresponden a personas que provienen de grupos sociales marginados. En la mayoría de los casos se trata de población que abandonó la escuela en el ciclo primario, repitiendo el patrón observado en sus familias. Tal itinerario educativo da cuanta de los obstáculos que aún deben superarse para romper con el círculo intergeneracional de la pobreza. Además, desde el punto de vista laboral, como se ha observado, la mayoría de los beneficiarios de la modalidad regular declaran no encontrarse trabajando o estar en una situación laboral precaria. Esta condición educativa limita el acceso al mercado laboral chileno que privilegia a los ciudadanos con un mejor capital cultural y con una certificación escolar. Tal como se ha determinado previamente, el desempleo y el empleo precario asociado a bajas remuneraciones pueden llegar a afectar a estos estudiantes desde el punto de vista del cumplimiento de sus responsabilidades y roles que la sociedad le asigna a la persona adulta. 
Desde el punto de vista discursivo, ciertamente existe una voluntad en los estudiantes por concluir sus estudios y proseguir estudios post secundarios. En los relatos de los estudiantes se percibe que los motivos para concluir la etapa escolar responden tanto a factores instrumentales como de realización personal. Por una parte, existe un grupo de estudiantes que busca obtener una certificación que les emita insertarse en el mundo laboral. Por otra parte, otro grupo accede a los cursos de educación de adultos asumiendo el desafío de mejorar su autoestima. En este último sentido, la re-escolarización se vincula de manera fundamental con la autoestima y la autorrealización de los sujetos - especialmente para los estudiantes de mayor edad - y no debe ser vista exclusivamente como un medio para obtener un mejor trabajo y, por ende, remuneraciones más altas. Las motivaciones que empujan a las personas a retomar sus estudios escolares responden a una mezcla de ambos factores de acuerdo con la evidencia.

Sin perjuicio de lo anterior, desde el punto de vista de las percepciones y relatos de los estudiantes EPJA, es posible sostener que tanto el fracaso escolar en el sistema formal como su experiencia en los cursos EPJA, la asocian a desempeños individuales o personales, más que a factores de contexto o situacionales. Lo relevante de este hallazgo, en el contexto chileno, radica en dos aspectos fundamentales: la existencia de un modelo de desarrollo económico (de carácter neoliberal) que ha promovido en los últimos 30 años el emprendimiento personal por sobre el colectivo; y, la reconstrucción individual que los estudiantes de EPJA le atribuyen a su itinerario educativo caracterizado por permanente fracaso. En otras palabras, el fracaso escolar es percibido por los estudiantes como un acto de irresponsabilidad personal y no es visibilizado también como una omisión de un sistema educativo que no ha garantizado el derecho a la educación. Con esta esta última frase se quiere decir que, se percibe una tendencia en el sentido que el fracaso educativo es visto como una cuestión personal, pero tal como se evidencia en el presente trabajo, los motivos asociados al fracaso educativo son multicausales, incluyendo por cierto factores internos (intra institucionales) y externos (extra institucionales).

\section{REFERENCIAS}

Bourdieu, P. Capital cultural, escuela y espacio social. México, DF: Siglo XXI, 1997.

Bryк, A.; Thum, Y. M. The effects of high school organization on dropping out: an exploratory investigation. American Educational Research Journal, London: Sage, v. 26, n. 3, p. 353-383, 1989.

Cairns, R.; Cairns, B.; Neckerman, H. Early school dropout: configurations and determinants. Child Development, Chichester, United Kingdom: The Society for Research in Child Development v. 60, n. 6, p. 1.437-1.452, 1989.

Castells, M. Redes de esperanza e indignación. Madrid: Alianza Editorial, 2012.

Castillo, D. Desertores de la educación básica. Reflexiones e interrogantes a partir de la práctica. Revista Latinoamericana de Innovaciones Educativas, Argentina: Ministerio de Cultura y Educación, n. 37, p. 69-90, 2003. 
Castillo, D.; Espinoza, O.; Gonzalez, L. E. Deserción en la educación primaria: una mirada desde la perspectiva de la equidad y la cohesión social. In; Espinoza, O.; Castillo, D.; González, L. E. (Eds.). Políticas públicas para fomentar la equidad y la cohesión social en el sistema educacional: escenarios, dilemas y desafíos. Santiago: LOM Ediciones, 2013. p. 68-88.

Comisión Intersectorial de Reinserción Educativa. Programa intersectorial de re-escolarización: construyendo alternativas educativas para niños, niñas y adolescentes en situación de vulnerabilidad. Santiago: UNESCO, 2006. Disponible en: <https:// es.scribd.com/document/103369847/Cuadernos-Del-Foro-Desercion-Escolar-enChile>. Acceso en: 30 enero 2015.

Croninger, R.; Lee, V. Social capital and dropping out of high school: benefits to at-risk students of teachers' support and guidance. Teachers College Record, New York: Columbia University, v. 103, n. 4, p. 548-581, 2001.

Espíndola, E.; León, A. La deserción escolar en América Latina: un tema prioritario para la agenda regional. Revista Iberoamericana de Educación, Madrid: OEI, n. 30, p. 39-62, 2002.

Espinoza, O.; Castillo, D.; Gonzalez, L. E. (Eds.). Politicas públicas para fomentar la equidad y la cohesión social en el sistema educacional: escenarios, dilemas y desafíos. Santiago: LOM Ediciones, 2013.

Espinoza, O.; Castillo, D.; Gonzalez, L. E. Perfiles de estudiantes de las modalidades de educación de adultos: una aproximación desde las cifras. Documento de Trabajo PIIE, Santiago: Programa Interdisciplinario de Investigaciones em Educación (PIIE), n. 1, p. 1-40, 2013a.

.;________ . Perfiles de estudiantes de las modalidades de educación de adultos: percepciones y valoraciones. Documento de Trabajo PIIE, Santiago: Programa Interdisciplinario de Investigaciones em Educación (PIIE), n. 2, p. 1-62, 2013b.

;_________ Caracterización de programas de reinserción escolar y de nivelación de estudios en Chile: modalidad regular. Documento de Trabajo PIIE, Santiago: Programa Interdisciplinario de Investigaciones em Educación (PIIE), n. 3, p. 1-6, 2013c. Espinoza, O.; Castillo, D.; Gonzalez, L. E.; Santa Cruz, J. C. Educación de adultos e inclusión social en Chile. Revista Psicoperspectivas, Valparaíso: PUCV, v. 13, n. 3, p. 69-81, 2014a. Disponible en: <http://www.psicoperspectivas.cl/index.php/ psicoperspectivas/article/viewFile/393/375>. Acceso en: 15 enero 2015.

Espinoza, O.; Loyola, J.; Castillo, D.; González, L. E. La educación de adultos en Chile: experiencias y expectativas de los estudiantes de la modalidad regular. Última Década, Valparaíso: CIDPA, n. 40, p. 159-181, jul.2014b.

Espinoza, O.; Gonzalez, L. E.; Loyola, J. Factores familiares asociados a la deserción escolar en Chile. Revista de Ciencias Sociales, Maracaibo: Universidad de Zulia, v.XVIII, n. 1, p. 136-150, 2012.

Espinoza, O.; Castillo, D.; González, L. E.; Santa Cruz, E.; Loyola, J. Fatores intraescolares associados ao abandono escolar no Chile: um estudo de caso. Revista Lusófona de Educacao, Lisboa: Universidad Lusófona de Humanidades y Tecnologías, n. 20, p. 31-48, 2012. 
Espinoza, O.; Castillo, D.; Gonzalez, L. E.; Loyola, J. Discusión teórica en torno a los determinantes de la deserción escolar. Documento de Trabajo CIE n. 3. Santiago: Universidad UCINF, 2010. Disponible en: <http://www.cie-ucinf.cl/ download/documentos_de_trabajo/CIE_doc_discusion_teorica.pdf $>$. Acceso en: 20 enero 2015. ;_________________ Estudiantes vulnerables y sus itinerarios educativos en el sistema escolar municipal en Chile. Revista Iberoamericana de Educación, Madrid: OEI, n. 60/4, p. 1-16, 2012.

Goldschmidt, P.; Wang, J. When can schools affect dropout behavior? A longitudinal multilevel analysis. American Educational Research Journal, London: Sage, v. 36, n. 4, p. 715-738, 1999.

Ingrum, A. High school dropout determinants: the effect of poverty and learning disabilities. The Park Place Economist, Bloomington, Illinois: Illinois Wesleyan University, v. 14, p. 73-79, 2007.

Janosz, M.; Leblanc, M.; Boulerice, B.; Tremblay, R. Disentangling the weight of school dropout predictors: a test on two longitudinal samples. Journal of Youth and Adolescence, New York: Springer, v. 26, n. 6, p. 733-762, 1997.

Knowles, M. The modern practice of adult education. Englewood Cliffs: Prentice Hall/ Cambridge, 1980.

Leenders, H.; Veugelers, W. Different perspectives on values and citizenship education. In: ZAJDA, J.; DAUN, H.(Eds.). Global values in education. Teaching democracy and peace. New York: Springer, 2009. p. 21-34.

MacNeal, R. Extracurricular activities and high school dropouts. Sociology of Education, London: Sage, v. 68, n. 1, p. 62-80, 1995.

Are students being pulled out of high school? The effect of adolescent employment on dropping out. Sociology of Education, London: Sage, v. 70, n. 3, p. 206220, 1997.

Marshall, T. Algunos factores que explican la deserción temprana. In: Conace. Abriendo calles: prevención del consumo de drogas en niños, niñas y adolescentes en situación de calle. Santiago: CONACE, 2003. p. 15-25.

Merriam, S. B.; Brockett, R. The profession and practice of adult education. San Francisco: Jossey-Bass, 1996.

Merriam, S. B.; Brockett, R. The profession and practice of adult education: an introduction. San Francisco: John Wiley \& Sons, 2007.

Mideplan. Educación: encuesta CASEN 2011. Santiago: MIDEPLAN, 2011.

Miкe, I.; NaKAJjo, A.; IsoKe, D. Socioeconomic determinants of primary school dropout: the logistic model analysis. Kampala, Uganda: Economic Policy Research Centre, 2008. (Research Series, n. 54).

Mineduc - Ministerio de Educación. Indicadores de la educación en Chile 20072008. Santiago: MINEDUC, Departamento de Estudios y Desarrollo de la División de Planificación y Presupuesto, 2008. 
Mineduc - Ministerio de Educación. Guía Ayuda MiNEDUC. Educación de adultos. Santiago: MINEDUC, 2012. Disponible en: <https://www.google.cl/ url $? \mathrm{sa}=\mathrm{t} \& \mathrm{rct}=\mathrm{j} \& \mathrm{q}=\& \mathrm{esrc}=\mathrm{s} \&$ source $=w e b \& \mathrm{c} d=2 \& \mathrm{cad}=\mathrm{rja} \& u a c t=8 \& v e d=0 \mathrm{ahUKE}$ wiY5Y6yzc_XAhVBkpAKHRXvCRQQFggzMAE\&url=http\%3A\%2F\%2 Fsd0090916c5a1c16a.jimcontent.com\%2Fdownload\%2Fversion\%2F1354574978\%2 Fmodule\%2F6281412582\%2Fname\%2Fguia_adul.pdf\&usg=AOvVaw0VguP5u0Z4pzd WP3ggY0RR >. Acceso en: 30 enero 2015.

. Orientaciones técnicas para la formulación de proyectos de reinserción escolar 2013. Santiago: MINEDUC, División de Educación General, Reinserción Escolar, 2013. OECD - Organization for Economic Co-operation and Development. Lifelong learning for all: policy directions. París: OECD, 2001.

. Lifelong learning: policy brief. París: OECD, 2004.

Ou, S.-R.; Mersky, J.; Reynolds, A.; Kohler, K. Alterable predictors of educational attainment, income, and crime: findings from an inner-city cohort. Social Service Review, Chicago: University of Chicago, v. 81, n. 1, p. 85-128, 2007.

Perreira, K.; Harris, K.; Lee, D. Making it in America: high school completion by immigrant and native youth. Demography, New York: Springer, v. 43, n. 3, p. 511-536, 2006.

Pomerantz, E.; Moorman, E.; Litwack, S. The how, whom, and why of parents' involvement in children's academic lives: More is not always better. Review of Educational Research, London: Sage, v. 77, n. 3, p. 373-410, 2007.

PREAL. Deserción escolar: un problema urgente que hay que abordar. Formas y Reformas de la Educación, Santiago: Programa de Promoción de la Reforma Educativa en América Latina y el Caribe, año 5, n. 14, p. 1-4, 2003. Disponible en: <http://hdrnet.org/78/1/ Desercion_Escolar.pdf $>$. Acceso en: 30 enero 2015.

Raczynski, D. Proceso de deserción escolar en la educación media. Factores expulsores y protectores. Santiago: Instituto Nacional de la Juventud, 2002.

Rotermund, S. Why students drop out of high school: comparison from three national surveys. Statistical brief 2. Califormia Dropout Research Project. Santa Barbara, CA: USA, 2007.

Rumberger, R. Dropping out of high school: the influence of race, sex, and family background. American Educational Research Journal, London: Sage, v. 20, n. 2, p. 199220, 1983.

. Dropping out of middle school: a multilevel analysis of students and schools. American Educational Research Journal, London: Sage, v. 32, n. 3, p. 583-625, 1995.

. Why students dropout of school and what can be done. Dropouts in America: how severe is the problem? What do we know about intervention and prevention? Boston: UCLA, enero 2001. Disponible en: < https://www.civilrightsproject.ucla.edu/ research/k-12-education/school-dropouts/why-students-drop-out-of-school-andwhat-can-be-done>. Acesso en: 2 Oct. 2017.

. Dropping out. Why students drop out of high school and what can be done about it. Boston: Harvard University Press, 2011. 
Rumberger, R.; Thomas, S. The distribution of dropout and turnover rates among urban and suburban high schools. Sociology of Education, London: Sage, v. 73, n. 1, p. 39-67, 2000.

Rumberger, R.; Lim, S. A. Why students drop out of school: a review of 25 years of research. California Dropout Research Project, Santa Barbara: Gevirtz Graduate School of Education, n. 5, 2008. p. 1-130.

Santos, H. Dinámica de la deserción escolar en Chile. Santiago: Departamento de Estudios División Social, MIDEPLAN, 2009. p. 1-25. Disponible en: <http://www. ministeriodesarrollosocial.gob.cl/btca/txtcompleto/mideplan/ser.estsoc-dinamdeserc. escolar.pdf >. Acceso en: 2 oct. 2017.

Sarrate, M. L. Educación de adultos: evaluación de centros y experiencias. Madrid: Narcea, 1997.

Schкоlniк, M.; Del Río, F. Trabajo infanto-juvenil y educación: diagnóstico de la realidad chilena. In: Seminario i 2 Años de Escolaridad: un requisito para la EQUidAd en Chile, Santiago, marzo, 2002. (Ponencia)

Schuller, T.; Watson, D. Learning through life. London, UK: NIACE, 2009.

Smith, M. Adult education. In: The Encyclopedia of Informal Education. London: INFED, 1999. Disponible en: <http://www.infed.org/thinkers/et-knowl. htm>. Acceso en: 28 de enero de 2015.

Sousa, B. Reinventar la democracia, reinventar el estado. Madrid: Sequitur, 2008.

Unesco. Resoluciones. In: Conferencia General de la Unesco, 19., 1976, Nairobi. Actas... Nairobi, 26 oct. a 30 nov. 1976. Disponible en: <http://unesdoc.unesco.org/ images/0011/001140/114038S.pdf>. Acceso en: 2 oct. 2017.

. Global report on adult learning and education. Hamburg, Germany: UIL, 2009a. . Experiencias educativas de segunda oportunidad. Investigación iluminativa. Plataforma Educativa MERCOSUR Chile-Uruguay. UNESCO/OREALC, 2009b. In: <http://www.innovemosdoc.cl/diversidad_equidad/investigacion_estudios/ CHILEURUGUAY.pdf>.

Veugelers, W. Education and humanism. Rotterdam: Sense Publishers, 2011.

Warren, J. R.; Cataldi, E. A historical perspective on high school students' paid employment and its association with high school dropout. Sociological Forum, New Jersey, Eastern Sociological Society, v. 21, n. 1, p. 113-143, 2006.

Warren, J.; LeE, J. The impact of adolescent employment on high school dropout: differences by individual and labor-market characteristics. Social Science Research, United Kingdom: Elsevier, v. 32, n. 1, p. 98-128, 2003.

Yin, Z.; Moore, J. Re-examining the role of interscholastic sport participation in education. Psychological Reports, London: Sage, v. 94, n. 3c, p. 1.447-1.454, 2004.

Zoellick, E. Philosophical orientation to adult learning: a descriptive study of Minnesota environmental education practitioners. Tesis (Maestría) - University of Minnesota Duluth, Estados Unidos, 2009. 


\section{SOBRE LOS AUTORES}

Oscar Espinoza Diaz es doctor en política, planificación y evaluación en educación por la University of Pittsburgh (Estados Unidos). Profesor de la Universidad Diego Portales (Chile).

E-mail: oespinoza@academia.cl

Dante Castillo Guajardo es doctor en educación por la Universidad Academia de Humanismo Cristiano (Chile). Profesor de la Universidad Diego Portales (Chile).

E-mail: dcastillo@estudiospiie.cl

Luis Eduardo González Fiegehen es doctor en planificación y administración educacional por la Harvard University (Estados Unidos). Profesor de la Universidad Diego Portales (Chile).

E-mail:1gonzalez@cinda.cl 